\title{
TAUOLA, TAUOLA universal interface PHOTOS and MC-TESTER: Status Report
}

\author{
Z. Waुs ${ }^{\mathrm{a} *}$ \\ anstitute of Nuclear Physics, Polish Academy of Sciences, \\ ul. Radzikowskiego 152, 31-342 Cracow, Poland
}

The status of Monte Carlo programs for the simulation of $\tau$-lepton production and decay in high-energy accelerator experiments is reviewed. No basic changes in the organization of the programs were necessary since the previous $\tau$-lepton conference in 2006. Minor in size, but practical, extensions for the use of the programs in high precision mixed languages enviromens are being introduced: (i) For the TAUOLA Monte Carlo generator of $\tau$-lepton decays, automated and simultaneous use of many versions of form-factors for the calculation of optional weights for fits was discussed. A pilot example of its use was presented. (ii) New tests for PHOTOS Monte Carlo for QED bremsstrahlung in $W$ decays were shown. (iii) Prototype version of the TAUOLA universal interface based on HepMC (the C++ event record) was mentioned. Its tests with the help of MC-TESTER were discussed.

Presented results illustrate the status of the projects performed in collaboration with Vladimir Cherepanov, Nadia Davidson, Piotr Golonka, Gizo Nanava, Tomasz Przedziński Elżbieta Richter-Was and others.

Presented at International workshop on Tau Lepton Physics, TAU08 Novosibirsk, Russia September, 2008

preprint IFJPAN-IV-2008-10

\section{Introduction}

The TAUOLA package [1/2/3/4] for the simulation of $\tau$-lepton decays and PHOTOS [5]6 for the simulation of radiative corrections in decays, are computing projects with a rather long history. Written and maintained by well-defined authors, they nonetheless migrated into a wide range of applications where they became ingredients of complicated simulation chains. As a consequence, a large number of different versions are presently in use. From the algorithmic point of view, they often differ only in a few small details, but incorporate many specific results from distinct $\tau$-lepton measurements or phenomenological projects. Such versions were mainly maintained (and will remain so) by the experiments taking precision data on $\tau$ leptons. On the other hand, many new applications were developed recently, often requiring a program interface to other pack-

\footnotetext{
* Supported in part by the Polish Government grant N202 06434 (2008-2010) and EU-RTN Programme, Contract No. MRTN-CT-2006-035482, "Flavianet".
}

ages (e.g. generating events for LHC, LC, Belle or BaBar physics processes). The programs structure, prepared for the convenience of users, was presented during previous $\tau$ conferences, and we will not repeat it here.

This time, let us concentrate on physics oriented results and special techniques being developped and used for fits, over the years. They may be worth some attention now, when high precision, high statistic data are being analyzed. FORTRAN to C++ migration is not as simple as one may think. On one hand it is generally believed that large projects are easier to maintain in more modern programming frameworks. On the other hand, FORTRAN software can not be abandoned. It usually carries a lot of physics expertise. It is also impractical to replace the software environment of mature experiments with something new. Fortunately, co-existence of the two languages is not a problem, at least not from the software point of view.

Our presentation is organized as follows. Sec- 
tion 2 is devoted to the discussion of optional weights in TAUOLA and their use for fits to the data already at the level of comparison with raw data. In section 3 we present some new results for the simulation with PHOTOS of radiative corrections in decays. Section 4 is devoted to MC-TESTER; the program which can be used for semi-automatic comparisons of simulation samples originating from different programs. The prototype, C++ based, TAUOLA universal interface is mentioned in that Section too.

Because of the limited space of the contribution, and sizable amount of other physically interesting results, some of them will be excluded from conference proceedings. Let us hope that they will find place in future works, possibly with collaborators mentioned in the Abstract. For these works, the present paper may serve as an announcement.

\section{Optional weights in TAUOLA Monte Carlo}

Physics of $\tau$ lepton decays requires sophisticated strategies for the confrontation of phenomenological models with experimental data. On one side high statistics experimental samples are collected, and the obtained precision is high, on the other hand, there is a significant crosscontamination between distinct $\tau$ decay channels. Starting from a certain precision level all channels need to be analyzed simultaneously. Change of parametrization for one channel contributiong to the background may be important for the fit of another one. This situation leads to a complex configuatin where a multitude of parameters needs to be simultaneously confronted with a multitude of observables. One has to keep in mind that the models used to obtain distributionbs in the fits may require refinements or even substantial rebuilds. The isospin relation to distribution measured in $e^{+} e^{-}$collistions at low energies offer additional control, but may be the source of even more complex systematic error considerations. Effects of QED bremsstrahlung need to be taken ito account as well. It may affect selection criteria and background contaminations in quite complex and unexpected ways.

From the statistical point of view it is best to resolve such system in one automated step using the method such as $[78$ for example. This can be of course very dangerous from the point of view of systematic error control. We will return to this point later. At this moment let us discuss necessary adaptations for TAUOLA for such methods to be used. In fact the principle of the changes is quite simple. The method is in use since long time and in different situations, see eg. 9. It is enough, to calculate, for each generated event (for each present in it decay of $\tau^{+}$and $\tau^{-}$separately) alternative weights; the ratios of matrix element squared obtained with new currents provided by the user, and the one actually used in generation are then the vector of weights. If the currents can be represented as a linear combination of elementary ones, then the weight for arbitary values of coefficients is a bilinear form in the relative coefficient:1 1 . In more general cases, linearization is necessary, and the whole procedure needs to be repeated with the currents obtained at one step used for generation at the next one. In this way, effects of the new currents will migrate to spin correlations between $\tau^{+}$and $\tau^{-}$as well.

The first example of the use of the method, is demonstrated in fig. 1. At this step of the work no detector effects were included. Experimental data were provided as a one-dimensional histogram. Simulation of $\tau$ production process was not performed. One can see, that the theoretical model provides double peak which is absent in the data. This is because of an interplay between intermediate state resonance parametrization and phase space jacobian. The same parametrization was used for 3 -scalar and 2-scalar systems. This points to the necessity of further investigations of unitarity constraints on the running width or better control of the data.

Options how to handle, in experimental software, information for weight calculation is under study now. In principle, information stored in the event record should be enough. This may not be a perfect match in all cases. For example, effects of the boost from laboratory frame to rest-frame of $\tau$ lepton and back, may result in

\footnotetext{
${ }^{1}$ We have applied such method in one of our other programs 9] for the studies of anomalous couplings of $Z$ and LEP data.
} 


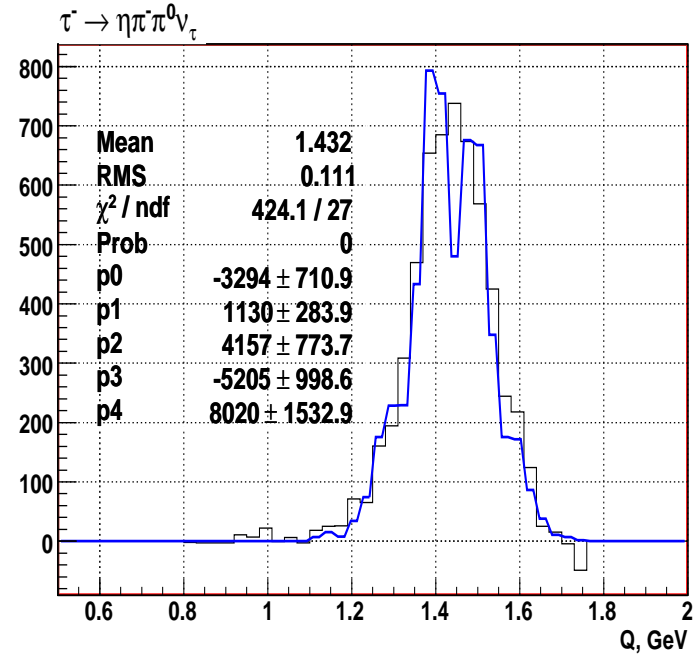

Figure 1. Preliminary result from the study of $\tau^{-} \rightarrow$ $\eta \pi^{-} \pi^{0} \nu$ decay using external weight. Experimental data and theoretical predictions obtained at the intermediate step of the work are shown. The distribution of the invariant mass of all final state hadrons is given. This plot is available thanks to courtesy of Vladimir Cherepanov.

rounding errors. In some cases the kinematical configuration may be changed because of QED bremsstrahlung generated by PHOTOS. The order of final state particles stored in the event record may not be the same as used internally in TAUOLA at the event construction step. This of course can be cross checked on the basis of inspection of a smaller sample and without detector simulation part being used. Such tests are nonetheless necessary, but may need less time than would be the case if collaboration software would be rebuild. From that point of view, the easiest would be to calculate optional weights from information already available in experiments production tapes, and only for those events where bremsstrahlung effects are not generated at all. This can be correted at the next step of form-factor iteration. Related slow down of the convergence should not be large. Possibly two steps will be enough, the second one for the verification. It will be necessary for control of spin correlations between the two decaying $\tau$-s as well. It is technically much simpler not to include changes into spin correlations. In such a case the vector of weights would depend on kinematical configurations of $\tau^{+}, \tau^{-}$and of the production process simultaneously. The optimal choice require further interaction with collaborations. Technical side of the activity will depend on the way the user want to calculate the hadronic current. It can be easily calculated as external generalized function communicating with TAUOLA at the level of linker or through the fifo pipes. They can be provided as FORTRAN or eg. C++ code and have well defined and easy to control input/output for modifications introduced into TAUOLA.

Int the past, and for a good reason, significant effort was devoted to strategies where the methotds as indicated here can be avoided. For example in 10] a system of projections was proposed for the 3 -scalar $\tau$ decays. If such a method can be used it is possible to obtain from the data just one of the contribution to the current, which is then parametrized by a single scalar function of two invariants only. This is of course on the expense of statistical strength on the measurement and abstraction of experimental sytematic error. Such methods, easy to apply for final states of two or three scalars, are less practical for higher multiplicities. Nonetheless they can be of great help to control the analysis and to verify which aspects of the models require improvements.

\section{PHOTOS and NLO effects in $W$ and $\gamma^{*} \rightarrow$ $\pi^{+} \pi^{-}$decays}

The changes introduced over the last two years into PHOTOS Monte Carlo program itself were rather small. On the other hand, the complexity of the work on its theoretical foundation was large and matches neither size nor the main purpose of the present talk (some of the related topics are revieved in [11). Previous tests of two body decays of the $Z$ into a pair of charged leptons [12] and scalar $B$ into a pair of scalars [13] was recently supplemented [14] with the study of $W^{ \pm} \rightarrow l^{ \pm} \nu \gamma$. The study of the process for $\gamma^{*} \rightarrow \pi^{+} \pi^{-}$is ongoing [15. In all of these cases universal kernel of PHOTOS was replaced with the one matching exact first order matrix element. In this way terms for 
NLO/NLL level are implemented. As usual, algorithm covers the full multiphoton phase-space and is exact in the infrared region of the phase space. This is rather unusual for the NLL compatible algorithms. One should not forget that PHOTOS generates weight one events and does not require any factorization scales. There is full overlap of the phase space where hard matrix element is used with the one for iterative photon emissions. All interference effects are implemented with the help of internal weights.

The results of all tests of PHOTOS with a NLO kernel are at sub-permille level. No differences with benchmarks can be observed, even for samples of $10^{9}$ events. For the comparisons when simpler physics assumptions are used, differences were sub-permille too, if compared to total rates (or were matching precision of programs used for tests).

This is very encouraging, and points to the possible extension of the approach outside of the QED (scalar QED) only. In particular to the domain of QCD or if phenomenological lagrangians for interactions of photons need to be applied. For that work to be completed spin amplitudes need to be further studied. Let us point here to the paper [16].

The discussed here refinements only indirectly affect practical side of simulations for $\tau$ physics. Changes in the kernels necessary for NLO may remain as options for tests only. They are available from the PHOTOS web page [14 but are not recomended for wider use. An example of such new tests is given in fig. $2 \mathrm{~b}$.

\section{MC-TESTER and $\mathrm{C}++$ TAUOLA universal interface}

In the development of packages such as TAUOLA or PHOTOS, question of tests and appriopriate relations to users applications are essential for their usefulness. In fact, users applications may be much larger in size and human effort than the programs discussed here. Good example of such 'user applications' are complete environments to fit experimental data and control detector uncertainties at the same time. As a consequence our programs need to work well with FORTRAN where
HEPEVT event record is used and C++ where HepMC [17] is used instead. For the sake of automatization of tests MC-TESTER was prepared [18]. Recently, new functionalities were introduced into the package [19. In particular, it works now with the HepMC event record, the standard of $\mathrm{C}++$ programs. The complete set-up for benchmarking the interfaces, such as interface between $\tau$-lepton production and decay, including QED bremsstrahlung effects is prepared. The example is chosen to illustrate the new options introduced into the program and novel ways of its use. From the technical perspective, [19] documents program updates and supplements previous documentation [18.

As in the past, test consists of two steps. Distinct Monte Carlo programs are run separately; events are searched for the decays of the chosen object, and information is stored by MC-TESTER. Then, at the analysis step, information from a pair of such runs may be compared and represented in the form of tables and plots.

One may have the impression that the modifications introduced into the release are minor and consist of simple improvements in the graphical representation of the output and purely technical reorganization thanks to the use of $\mathrm{C}++$ lists.

To some degree this is true, but on the other hand the changes were introduced because of pressure from applications. Let us show how program modifications can be used for non-trivial practical applications.

It is quite common that information stored in the event record is too large. For example individual soft photons which remain undetectable are present. Not only they do not influence the detector response at all, but they exhibit technical aspects of eg. infrared regulators of QED bremsstrahlung. In response, MC-TESTER should ignore those photons while analyzing decays (or group together with other particles). Otherwise, comparisons of different Monte Carlo programs would be dominated by the technical aspect of the implementation of the infrared regulator; MC-TESTER operation need to be adopted for this, see eg. 2012].

Another example where the event tree may need to be simplified for validation is if spin corre- 


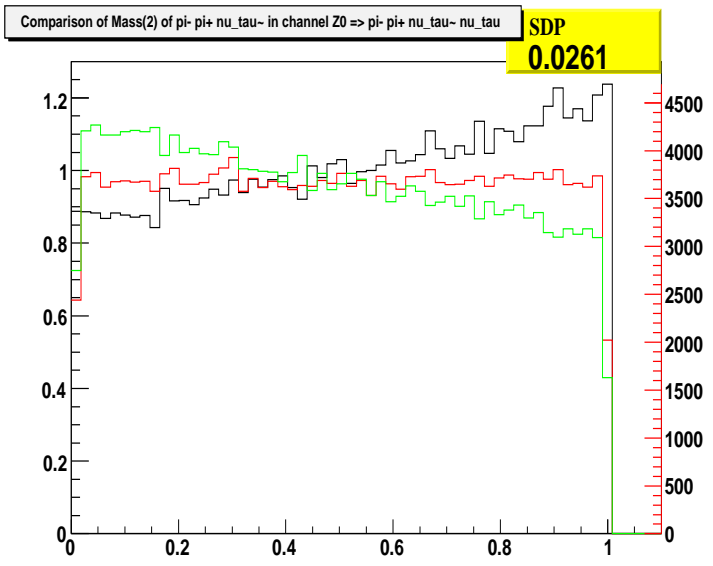

a

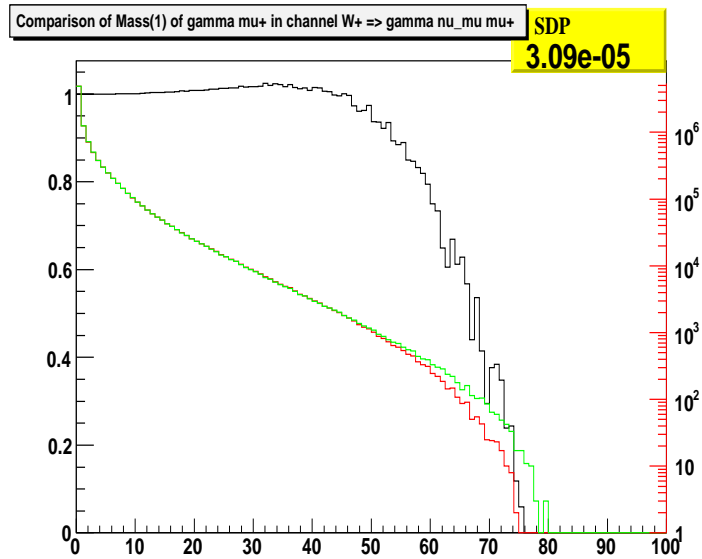

$\mathrm{b}$

Figure 2. Examples of interesting benchmarks: In the decay $Z \rightarrow \tau^{+} \tau^{-} \rightarrow \pi^{+} \pi^{-} \nu \bar{\nu}$, the $\pi^{-}$energy spectrum in the $Z$ rest-frame has identical shape as the distribution of the invariant mass squared of $\pi^{+} \pi^{-} \bar{\nu}$, see plot a. This linear distribution can be used to measure decaying $\tau^{-}$polarization. In plot $b$, an example of the PHOTOS test is given. Program version with full matrix element for $W^{+} \rightarrow \mu^{+} \nu_{\mu} \gamma$ channel is compared with the standard one. The distribution of the $\mu^{+} \gamma$ pair mass is shown.

lations are appropriately introduced into various production processes. Let us use as an example 2 $p p \rightarrow Z / \gamma *+X, Z \rightarrow \tau^{+} \tau^{-}$. It is convenient to start the test by restricting $\tau$ decays to the simplest decay mode, that is $\tau^{ \pm} \rightarrow \pi^{ \pm} \nu$, and look at distributions in cascade decay $Z \rightarrow \pi^{+} \pi^{-} \nu \bar{\nu}$. In this case the effects of spin correlations are largest. The distribution of the $\pi^{-}$energy spectrum (in the $Z$ rest-frame), manifests the $\tau$ polarization through its slope (see fig 2 a). Fortunately, this frame dependent spectrum is equal to the distribution of the square of invariant mass of $\pi^{-} \pi^{+} \bar{\nu}$. This distribution can be obtained in MC-TESTER thanks to the new options.

Final state activities will lead to final states of $Z$ decays where the $\tau$-pair is accompanied by bremsstrahlung photons or soft hadrons. Such distribution are usually strongly peaked. In fig. $2 \mathrm{~b}$ we provide an example of such a distribution but for the comparison of PHOTOS with exact marix element for the decay $W^{+} \rightarrow \mu^{+} \nu \gamma$.

With the above brief examples we have demonstrated that MC-TESTER may be useful for tests of libraries of particles decays, as well as for tests of their interfaces. The work on HepMC based TAUOLA universal interface is on-going. A preliminary version is already available [21, but from physics point of view it is still less complete than the FORTRAN version 22 .

The updated version of MC-TESTER was found 2012 to handle well cases where physically spurious information (eg. on soft photons) need to be ignored.

\section{Summary and future possibilities}

The status of the computer programs for the decay of $\tau$ leptons TAUOLA and associated projects TAUOLA universal interface and MC-TESTER was reviewed. The high-precision version of PHOTOS for radiative corrections was presented too.

New results for PHOTOS and decay of $W$ were mentioned. For this channel complete next-toleading order effects can now be simulated. However, for most of the applications these effects are not necessary, leaving the standard modu-

${ }^{2}$ Spin correlations in decays of $W, H, H^{ \pm}$into $\tau$ lepton(s) are nearly identical. 
lar version of PHOTOS sufficient. This exercise is important not only for photonic bremsstrahlung. It helps to understand better questions related with matching hard emission matrix elements with parton showers without necessity to introduce any boundaries within phase space. The important result of the above work is that the path to include electromagnetic form-factors of the particles participating in decay is now open for future fits to the data. These form-factor effects may be significantly larger and physically more justified than complete next-to-leading order effects of scalar QED as in $B$ meson decays.

The presentation of the TAUOLA generalpurpose interface was brief. The C++ version exist, but is still physically less refined than the FORTRAN version. Examples for its use can be found on the continuously modified web page [21]. The new version of MC-TESTER [19] is already public now. It not only works with HepMC [17] of C++ but enables user defined tests.

Distinct versions of the TAUOLA library for $\tau$ lepton decay, and of PHOTOS for radiative corrections in decays, are now in use. The principles how to use the distribution package are presented in refs. 423], on the other hand, development of new and recovery of codes for old strategies of fits is becoming available. This includes strategy to use weighted events, and the projection operators [10 for the three scalars final states. Let us hope that the present talk will provide some stimulation for the larger work in the near future.

\section{Acknowledgements}

Discussions with members of the Belle and BaBar collaborations are also acknowledged. Exchange of e-mails and direct discussions with S. Banerjee, S. Eidelman, H. Hayashii, K. Inami, A. Korchin, J. H. Kühn and O. Shekhovtsova was a valuable input to present and future steps in project development.

\section{REFERENCES}

1. S. Jadach, J. H. Kuhn, and Z. Was, Comput. Phys. Commun. 64 (1990) 275.

2. M. Jezabek, Z. Was, S. Jadach, and J. H. Kuhn, Comput. Phys. Commun. 70 (1992)
69.

3. S. Jadach, Z. Was, R. Decker, and J. H. Kuhn, Comput. Phys. Commun. 76 (1993) 361-380.

4. P. Golonka et al., Comput. Phys. Commun. 174 (2006) 818-835, hep-ph/0312240.

5. E. Barberio, B. van Eijk, and Z. Was, Comput. Phys. Commun. 66 (1991) 115.

6. E. Barberio and Z. Was, Comput. Phys. Commun. 79 (1994) 291-308.

7. A. Hoecker, P. Speckmayer, J. Stelzer, F. Tegenfeldt, H. Voss, K. Voss, et al., http://tmva.sourceforge.net/

8. A. Hocker et al., PoS ACAT (2007) 040, physics/0703039.

9. A. Jacholkowska, J. Kalinowski, and Z. Was, Comput. Phys. Commun. 124 (2000) 238242, hep-ph/9905225.

10. J. H. Kuhn and E. Mirkes, Z. Phys. C56 (1992) 661-672.

11. Z. Was, Acta Phys. Polon. B39 (2008) 1761, 0807.2775 .

12. P. Golonka and Z. Was, Eur. Phys. J. C50 (2007) 53-62, hep-ph/0604232.

13. G. Nanava and Z. Was, Eur. Phys. J. C51 (2007) 569-583, hep-ph/0607019.

14. P. Golonka, G. Nanava, and Z. Was, Tests of PHOTOS Hard Bremsstrahlung, http://mc-tester.web.cern.ch/MC-TESTER/PHOTOS-MCTE

15. Z. Was and Q. Xu, In preparation.

16. A. van Hameren and Z. Was, 0802.2182.

17. M. Dobbs and J. B. Hansen, Comput. Phys. Commun. 134 (2001) 41-46, https://savannah.cern.ch/projects/hepmc/.

18. P. Golonka, T. Pierzchala, and Z. Was, Comput. Phys. Commun. 157 (2004) 39-62, hep-ph/0210252.

19. N. Davidson, P. Golonka, T. Przedzinski, and Z. Was, 0812.3215.

20. P. Golonka and Z. Was, Eur. Phys. J. C45 (2006) 97-107, hep-ph/0506026

21. N. Davidson, T. Przedzinski, E. Richter-Was, and Z. Was, Presently the code is evolving quickly. Interested pilot users can download code from http://www.ph.unimelb.edu.au/ ndavidson/tauola/doxygen/index.html It is automatically updated daily from our source code repository. Version information can be found in a text file contained in the distribu- 
tion.

22. T. Pierzchała, E. Richter-Wạs, Z. Wąs, and M. Worek, Acta Phys. Polon. B32 (2001) 1277-1296, hep-ph/0101311.

23. Z. Was and P. Golonka, Nucl. Phys. Proc. Suppl. 144 (2005) 88-94, hep-ph/0411377. 